\title{
An Elastic Half Space with a Moving Punch
}

\author{
SANDIP SAHA ${ }^{1, *}$, VIKASH KUMAR ${ }^{1}$, APURBA NARAYAN DAS ${ }^{2}$ \\ ${ }^{1}$ Department of Mathematics, Madanapalle Institute of Technology And Science, Andhra Pradesh- \\ 517325, INDIA \\ ${ }^{2}$ Department of Mathematics, Alipurduar University, Alipurduar, West Bengal-736121, INDIA
}

\begin{abstract}
The dynamic problem of a punch with rounded tips moving in an elastic half-space in a fixed direction has been considered. The static problem of determining stress component under the contact region of a punch has also been solved. Fourier integral transform has been employed to reduce the problems in solving dual integral equations. These integral equations have been solved using Cooke's [1] result (1970) to obtain the stress component. Finally, exact expressions for stress components under the punch and the normal displacement component in the region outside the punch have been derived. Numerical results for stress intensity factor at the punch end and torque applied over the contact region have been presented in the form of graph..
\end{abstract}

Key-Words: - Punch, Fourier transform, Integral equations, Stress intensity factor, Torque

Received: April 20, 2021. Revised: October 14, 2021. Accepted: November 2, 2021. Published: November 22, 2021.

\section{Introduction}

Generally, contact problems occur in mechanical engineering, civil engineering, and materials science. Different structures are formed as a concrete foundation planted below the ground surface and contacts on large scale take place between the foundation and deformable ground. Contacts on small scale appear in small-scaled indentation tests in the field of mechanical engineering, material science. The details of such penetration may be considered to study the stress distribution under the indenter, the surface displacements, the contact site, etc. Such analysis has become very much helpful for designing in geotechnical and footing engineering or the characterization of maters via indentation tests.

Galin [1] and Gladwell [2] have discussed the punch problems within the theory of elasticity thoroughly in the books authored by them. Sve and Keer [3] considered the plane problem of two punches moving with uniform velocity over an elastic layer on a rigid foundation. Brock [4-6] studied several problems of symmetric and non-symmetric frictionless indentations over a uniformly expanding contact region using the method of homogeneous function. Using the complex variable method, Talit and Moode [7] solved the moving punch problems. Problems of two and four moving punches have also been solved using the integral transform technique. Singh and Dhaliwal [8] studied the problem of antiplane indentation of an elastic layer by two moving punches. The same problems with four moving punches were solved by Das [9]. Dalmeya et. al.
[10] investigated the problem of indentation of an adhesive elastic layer of film over a rigid substrate by a rigid cylindrical punch assuming that the Hertzian contact is coupled with the tools of fracture mechanics. They employed a semi-analytical method using the Chebyshev polynomials. A review of recent works on inclusions has been made by Zhou et.al. [11]. They provided a detailed survey of inclusions of infinite space, a semi-infinite space subjected to surface loading and surface contact loading, or of a finite space. Various methods of solving the problems of single inclusions, double inclusions, multiple inclusions, etc. have been discussed in their work in detail. Peijian et.al. [12] studied the contact behavior of a rigid cylindrical punch sliding arbitrarily over an elastic half-space with varying shear modulus using Fourier integral transform. In this case, the problem has been solved after reducing the problem to a singular integral equation of the second kind. Argatov and Sabina [13] considered the axisymmetric problem of unilateral frictionless indentation of a homogeneous elastic half-space. [14] Kala has discussed limit state of strucure Global Sensitivity Analysis. [15] Rubio et. al. studied heat transfer process with solid-solid interface using numerical solution. Burova [16] solved the local Polynomial and non-polynomial Splines problem using numerical solution of the Volterra integral equation of the second kind. Using the MATLAB program they determined the deformation of granular material under a smooth rigid wedge punch. Comez et. al. [17] considered the problem of sliding of a rigid cylindrical punch over an orthotropic layer over an isotropic half- 
space. The cylindrical punch is assumed to move with a constant velocity in the lateral direction. Using Fourier integral transform technique and Galilean transformation, the problem is reduced to a singular integral equation, which is solved using Gauss-Jacobi integral formula.

In this paper, both the dynamic and static problems of a punch with rounded tips have been solved employing the integral transform technique. Fourier integral transformations have been employed to reduce the problem in solving dual integral equations. Using Cooke's result [1], the integral equations have been solved to obtain the stress component under the punch and the normal displacement component in the region outside the punch. Finally, stress intensity factor and torque over the contact region have been computed and numerical results showing the variations in those with the velocity of the punch have been presented in the form of graphs.

\section{Formulation and solution of problem I}

We consider an isotropic, homogeneous and semiinfinite medium, $y \leq 0$. A punch with rounded tip is assumed to move steadily with a constant velocity $V$ in the positive $X$-direction on $Y=0$. The equations of motion (neglecting body force) in terms of displacements are

$$
\begin{aligned}
& (\lambda+2 \mu)\left[u_{, X X}+v_{, X Y}\right]+\mu\left[u_{, Y Y}-v_{, X Y}\right]= \\
& \rho u_{, T T} \\
& (\lambda+2 \mu)\left[u_{, X Y}+v_{, Y Y}\right]+\mu\left[v_{, X X}-u_{, X Y}\right]=\rho u_{, T T}
\end{aligned}
$$

where $u, v$ noted as the displacement components in $X, Y$ directions respectively, $\lambda, \mu$ are Lame's constants and $u_{, X}$ denotes the partial derivatives of $u$ with respect to $X$. For a punch moving with constant velocity $V$ in the $X$-direction it is convenient to introduce the Galilean transformation

$x=X-V t, y=Y, z=Z$ and $t=T$

with $x, y, z$ as the translating coordinate system as shown in figure 1 .

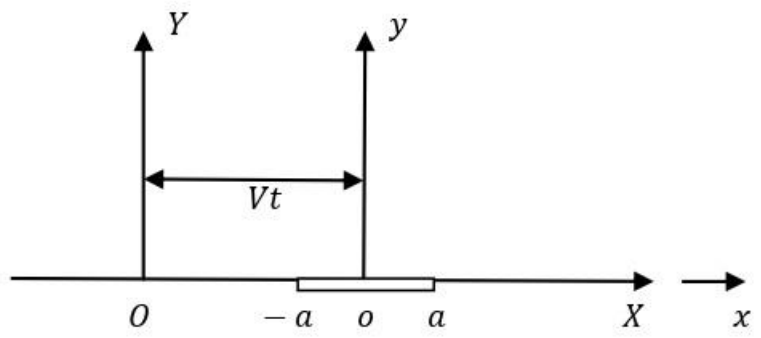

Fig. 1. Geometry and coordinate system

In the moving coordinates, the above equations of motion are reduced to

$$
\begin{aligned}
& \left(\lambda+2 \mu-\rho V^{2}\right) u_{, x x}+(\lambda+\mu) v_{, x y}+u_{, y y}=0 \\
& (\lambda+2 \mu) v_{, y y}+\left(\mu-\rho V^{2}\right) v_{, x x}+(\lambda+\mu) u_{, x y}=0
\end{aligned}
$$

Introducing,

$\bar{u}_{s}(\xi, y)=\int_{0}^{\infty} u(x, y) \sin (x \xi) d x$

$\int_{0}^{\infty} v(x, y) \cos (x \xi) d x$

$$
\bar{v}_{c}(\xi, y)=
$$

and

$\frac{2}{\pi} \int_{0}^{\infty} \bar{u}_{s}(\xi, y) \sin (x \xi) d x$ $u(x, y)=$

$\frac{2}{\pi} \int_{0}^{\infty} \bar{v}_{c}(\xi, y) \cos (x \xi) d x$

$$
v(x, y)=
$$

in the equations [4-5], we obtain the following form of equations

$\xi^{2}\left(\lambda+2 \mu-\rho V^{2}\right) \bar{u}_{s}+\xi(\lambda+\mu) \bar{v}_{c, y}-\mu \bar{u}_{s, y y}=0$

$(\lambda+2 \mu) \bar{v}_{c, y y}-\xi^{2}\left(\mu-\rho V^{2}\right) \bar{v}_{c}+$

$\xi(\lambda+\mu) \bar{u}_{s, y}=0$

Elimination of $\bar{u}_{s}$ from the equations (10-11) yields the following ODE

$\left[\begin{array}{c}\left\{\frac{d^{2}}{d y^{2}}-\left(1-M^{2} k^{2}\right) \xi^{2}\right\} \\ \left\{\frac{d^{2}}{d y^{2}}-\left(1-M^{2}\right) \xi^{2}\right\}\end{array}\right] \bar{v}_{c}=0$

with $\bar{v}_{c}(\xi, y) A(\xi) e^{-\xi y \sqrt{1-M^{2} k^{2}}}+$

$B(\xi) e^{-\xi y \sqrt{1-M^{2}}}$

Where unknown functions $A(\xi)$ and $B(\xi)$ are to be determined using the boundary conditions of the problem. Employing the result given by (13) in the equations $(10,11)$

$$
\begin{gathered}
\bar{u}_{s}(\xi, y)=\frac{A(\xi)}{\sqrt{1-M^{2} k^{2}}} e^{-\xi y \sqrt{1-M^{2} k^{2}}}+ \\
B(\xi) e^{-\xi y \sqrt{1-M^{2}}} \sqrt{1-M^{2}}, \mathrm{y} \geq 0
\end{gathered}
$$

Taking inverse transform we get from equations (13-14), we have

$$
\begin{gathered}
v(x, y)=\frac{2}{\pi} \int_{0}^{\infty}\left[A(\xi) e^{-\xi y \sqrt{1-M^{2} k^{2}}}+\right. \\
\left.B(\xi) e^{-\xi y \sqrt{1-M^{2}}}\right] \cos (x \xi) d \xi(15) \\
u(x, y)=\frac{2}{\pi} \int_{0}^{\infty}\left[\frac{A(\xi)}{\sqrt{1-M^{2} k^{2}}} e^{-\xi y \sqrt{1-M^{2} k^{2}}}+\right. \\
\left.B(\xi) e^{-\xi y \sqrt{1-M^{2}}} \sqrt{1-M^{2}}\right] \sin (x \xi) d \xi(16)
\end{gathered}
$$

Using the results given by (15-16) in the following formulae

$\sigma_{y y}=\lambda\left(u_{, x}+v_{, y}\right)+2 \mu v_{, y}$

$\sigma_{x y}=\lambda\left(u_{, y}+v_{, x}\right)$

we obtain 


$$
\begin{gathered}
\sigma_{y y}(x, y)= \\
-\frac{2 \mu}{\pi} \int_{0}^{\infty} \xi\left[\frac{\left(2-M^{2}\right) A(\xi)}{\sqrt{1-M^{2} k^{2}}} e^{-\xi y \sqrt{1-M^{2} k^{2}}}+\right. \\
\left.2 B(\xi) e^{-\xi y \sqrt{1-M^{2}}} \sqrt{1-M^{2}}\right] \cos \xi x d \xi \\
\sigma_{x y}(x, y)= \\
-\frac{2 \mu}{\pi} \int_{0}^{\infty} \xi\left[2 A(\xi) e^{-\xi y \sqrt{1-M^{2} k^{2}}}+(2-\right. \\
\left.\left.M^{2}\right) B(\xi) e^{-\xi y \sqrt{1-M^{2}}}\right] \cos \xi x d \xi
\end{gathered}
$$

Referred to the translating coordinates $(x, y, z)$, the punch is assumed to be located at $|x| \leq a, y=0$. So, the boundary conditions of the problem (on account of symmetry with respect to $x=0$ )

$v(x, 0)=-f(x), 0<x<a$

$\sigma_{y y}(x, 0)=0, x>a$

$\sigma_{x y}(x, 0)=0,0<x<\infty$

where $f(x)$ is an even function of $x$, lead to the following dual integral equation in $A(\xi)$

$$
\int_{0}^{\infty} A(\xi) \cos (x \xi) d \xi=\frac{\pi\left(2-M^{2}\right)}{2 M^{2}} f(x),
$$

$0 \leq x \leq a$

$\int_{0}^{\infty} \xi A(\xi) \cos (x \xi) d \xi=0, \quad x>a$

In order to solve the equations (24-25), we assume

$A(\xi)=\frac{1}{\xi} \int_{0}^{a} g(u) \cos (u \xi) d u$

Using the result

$\int_{0}^{a} \cos (u x) d x=\pi \delta(\xi)$

we see that the equation (25) is satisfied for any choice of $g(u)$. Substituting the result given by equation (26) in the equation (24) and differentiating both sides with respect to $x$, we get

$$
\int_{0}^{a} \frac{g(u)}{x^{2}-u^{2}} d u=
$$

$-\frac{\pi\left(2-M^{2}\right)}{2 M^{2}} \frac{f^{\prime}(x)}{x}, 0 \leq x \leq a$,

where the following results has been used $\int_{0}^{\infty} \sin (\xi x) d x=\frac{1}{\xi}$

After integrating the equation (28) with respect to $x$ from 0 to $x$, we get

$$
\begin{array}{r}
\int_{0}^{a} \frac{g(u)}{2 u} \ln \left|\frac{x+u}{x-u}\right| d u= \\
\frac{\pi\left(2-M^{2}\right)}{2 M^{2}} \int_{0}^{x} \frac{f^{\prime}(y)}{y} d y
\end{array}
$$

Using Cooks result [1], we obtain

$$
\begin{gathered}
g(u)= \\
\frac{2}{\pi} \frac{\left(M^{2}-2\right)}{M^{2}} \frac{u^{2}}{\sqrt{a^{2}-u^{2}}} \int_{0}^{a} \frac{\sqrt{a^{2}-y^{2}}}{y} \frac{f^{\prime}(y)}{y^{2}-u^{2}} d y
\end{gathered}
$$

Using the expression given by (31) in the result (19), the stress component under the punch is found as

$$
\left[\sigma_{y y}(x, 0)\right]_{0<x<a}=\frac{\mu R(M) g(x)}{\left(2-M^{2}\right) \sqrt{1-M^{2} k^{2}}}
$$

with $\quad R(M)=4 \sqrt{\left(1-M^{2} k^{2}\right)\left(1-M^{2}\right)}-$ $\left(2-M^{2}\right)^{2}$ and $g(x)$ is given by the equation (31).

The normal displacement component outside the contact region is given by

$$
\begin{aligned}
& {[v(x, 0)]_{x>a}=} \\
& -\frac{4}{\pi^{2}} \int_{a}^{x} \operatorname{tdt} \int_{0}^{a} \frac{u^{2}}{\sqrt{a^{2}-u^{2}}} \frac{d u}{\left(u^{2}-t^{2}\right)} \int_{0}^{a} \frac{\sqrt{a^{2}-y^{2}}}{y} \frac{f^{\prime}(y)}{y^{2}-u^{2}} d y
\end{aligned}
$$

Using the results

$$
\begin{gathered}
\int_{0}^{a} \frac{u^{2}}{\sqrt{a^{2}-u^{2}}} \frac{d u}{\left(u^{2}-t^{2}\right)\left(y^{2}-u^{2}\right)}= \\
-\frac{\pi t^{2}}{2 \sqrt{\left(t^{2}-a^{2}\right)\left(y^{2}-t^{2}\right)}}, \\
\int_{a}^{x} \frac{t^{2}}{\sqrt{t^{2}-a^{2}}} \frac{d t}{y^{2}-t^{2}}=-\cosh ^{-1} \frac{x}{a}- \\
\frac{y^{2}}{\sqrt{a^{2}-y^{2}}} \tan ^{-1} \frac{y \sqrt{x^{2}-a^{2}}}{x \sqrt{a^{2}-y^{2}}},
\end{gathered}
$$

we get

$$
[v(x, 0)]_{x>a}=
$$

$-\frac{2}{\pi}\left[\cosh ^{-1} \frac{x}{a} \int_{0}^{a} \sqrt{a^{2}-y^{2}} \frac{f^{\prime}(y)}{y} d y+\right.$

$\left.\int_{0}^{a} \tan ^{-1} \frac{y \sqrt{x^{2}-a^{2}}}{x \sqrt{a^{2}-y^{2}}} y f^{\prime}(y) d y\right]$

The stress intensity factor at the end of the punch is obtained using (31) and (32) as

$N=$
$\lim _{x \rightarrow a+} \sqrt{2(a-x)}\left[\sigma_{y y}(x, 0)\right]_{0<x<a}=$
$\frac{2 \mu R(M) a^{3 / 2}}{\pi M^{2} \sqrt{1-M^{2} k^{2}}} \int_{0}^{a} \frac{f^{\prime}(x) d x}{x \sqrt{a^{2}-x^{2}}}$

Using the result $\int_{0}^{a} \frac{x^{2} d x}{\sqrt{a^{2}-x^{2}}\left(y^{2}-x^{2}\right)}=-\frac{\pi}{2}, 0<y<a$ we get the torque applied over the contact region is obtained as

$$
\begin{array}{r}
T=-2 \int_{0}^{a} \sigma_{y y}(x, 0) d x= \\
\frac{2 \mu R(M)}{M^{2} \sqrt{1-M^{2} k^{2}}} \int_{0}^{a} \sqrt{a^{2}-x^{2}} \frac{f^{\prime}(x)}{x} d x
\end{array}
$$

It is to be noted that the stress component under the punch depends on the velocity of the moving punch, but in the plane of the punch the displacement component is independent of it.

\section{Formulation and solution of problem II}

Please, leave two blank lines between successive sections as here.

In this section, we consider a semi-infinite homogeneous, isotropic material with a punch located at $Y=0,|X| \leq a$. The equations of motion (neglecting body force) in terms of displacements are

$(\lambda+2 \mu)\left[u_{, X X}+v_{, X Y}\right]+\mu\left[u_{, Y Y}-v_{, X Y}\right]=0$
$(\lambda+2 \mu)\left[u_{, X Y}+v_{, Y Y}\right]+\mu\left[v_{, X X}-u_{, X Y}\right]=0$

where $u, v, \lambda, \mu$ have already been defined earlier.

Application of the same technique as that employed in the problem 1 and use of the conditions (on account of symmetry with respect to $X=0$ )

$$
\begin{array}{r}
V(X, 0)=-h(X) \quad 0 \leq X \leq a \\
\sigma_{y y}(X, 0)=0, \quad X>\mathrm{a}
\end{array}
$$




$$
\sigma_{x y}(X, 0)=0,0<X<\infty
$$

with $h(X)$ as an even function of $X$, lead to the dual integral equation in $\mathrm{C}(\xi)$ :

$\int_{0}^{\infty} C(\xi) \cos (\xi X) d \xi=-\frac{\pi}{2} h(X), \quad 0 \leq X \leq a$

$\int_{0}^{\infty} \xi C(\xi) \cos (\xi X) d \xi=0, \quad X>a$

It is to be mentioned that the above integral equations cannot be derived using the corresponding expressions of the dynamic problem given in the equations (24-25) on putting $M=0$. Now using the same method as that employed in problem I, we can easily show that

$$
\left[\sigma_{y y}(X, 0)\right]_{0<X<a}=-\frac{2 \mu(\lambda+\mu)}{(\lambda+2 \mu)} h(X)
$$

where

$$
\begin{gathered}
h(X)= \\
\frac{2}{\pi} \frac{X^{2}}{\sqrt{a^{2}-X^{2}}} \int_{0}^{a} \frac{\sqrt{a^{2}-y^{2}}}{y} \frac{f^{\prime}(y)}{\left(y^{2}-X^{2}\right)} d y
\end{gathered}
$$

The stress intensity factor at the end of punch is obtained as

$$
S=\frac{4 \mu(\lambda+\mu) a^{3 / 2}}{(\lambda+2 \mu)} \int_{0}^{a} \frac{h^{\prime}(t)}{t \sqrt{a^{2}-t^{2}}} d t
$$

The torque applied over the contact region is obtained as

$$
\begin{aligned}
& T=-2 \int_{0}^{a} \sigma_{y y}(X, 0) d X= \\
& -\frac{4 \mu(\lambda+\mu)}{(\lambda+2 \mu)} \int_{0}^{a} \sqrt{a^{2}-t^{2}} \frac{h^{\prime}(t)}{t} d t
\end{aligned}
$$

\section{Numerical results and discussions}

In this section, the numerical results for the problem I have been presented in the form of graph. The variation of stress intensity with $\frac{V}{c_{2}}$ for different values of $n$ taking $\lambda=\mu$ and $b_{k} a^{2 k-1}=1$, have been presented. As the velocity of the punch is less than Rayleigh wave velocity, it is reasonable to take the values of $M \leq 0.9194$. If we take $f(x)=$ $\sum_{k=1}^{n} b_{k} x^{2 k}$, then the results given by (34) and (35) simplified to

$$
\begin{array}{ll}
\frac{4 \mu R(M) a^{\frac{3}{2}}}{\pi M^{2} \sqrt{1-M^{2} k^{2}}} \sum_{k=1}^{n} k b_{k} I_{2 k-2}, &
\end{array}
$$

$$
\frac{-4 \mu R(M)}{M^{2} \sqrt{1-M^{2} k^{2}}} \sum_{k=1}^{n} k b_{k} J_{2 k-2}, \quad T=
$$

With $J_{2 k}=\frac{a^{2} I_{2 k}}{2 k+2}=\int_{0}^{a} t^{2 k} \sqrt{a^{2}-t^{2}} d t$,

$\int_{0}^{a} \frac{t^{2 k}}{\sqrt{a^{2}-t^{2}}} d t=\frac{(2 k-1)(2 k-3) \ldots \ldots .5 .3 .1}{2 k(2 k-2) \ldots \ldots .6 .4 .2} \frac{\pi a^{2 k}}{2}$

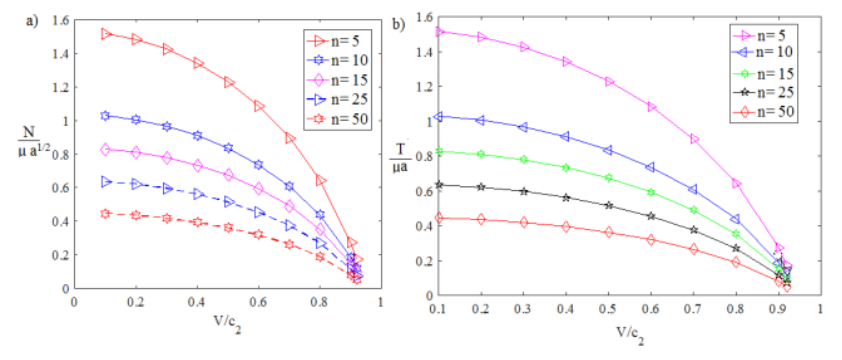

Fig. 2. Variations of Stress intensity factor (a) and torque (b) with normalized velocity.

From figure 2(a), we see that the values of the dimensionless stress intensity factor gradually decreases as $\frac{V}{c_{2}}$ increases as tends to 0 as $\frac{V}{c_{2}}$ tends to 0.9194 , as expected. It is also found from that graph that as the degree of the polynomial, i.e., $n$ increases, the value of stress intensity factor decreases. In figure 2(b), the variations in the torque over the contact region with $\frac{V}{c_{2}}$ have been depicted. It is found form those figures that the variations in dimensionless torque over the contact region and dimensionless stress intensity factor with $\frac{V}{c_{2}}$ and $n$ are of same type.

\section{Conclusion}

In most of the cases associated with punch problems, the normal component of the displacement along the contact regions is assumed as constant to avoid complexity in mathematical calculations. Generally, this is not always true as shape of the indenter may vary and rigidity of semiinfinite medium over which the indenter acts is not uniform. To the best knowledge of the authors, very few problems of indentation of an elastic half space by a moving punch associated with the normal component of the displacement as a function have been solved till now. In this work, we have assumed the normal component of the displacement along the contact region as a polynomial function of even degree (due to symmetry). The effect of the degree of the polynomial function and variations in velocity of the punch on the stress intensity factor at the punch end and also on the torque over the contact region has been studied. 


\section{References:}

[1] L. A. Galian, Contact problems in elasticity theory, Rayleigh: North Carolina Stage College, 1961.

[2] G. M. L. Gladwell, Contact problems in classical theory of elasticity, Sijth and Noordhooff, The Netharlends, 1980.

[3] C. Sve \& L. M. Keer, Indentation of elastic layer by moving punches. I. J. Solid Structure, 5, 795-816, 1969.

[4] L. M. Brock, Symmetrical frictionless indentation over a uniformly expanding contact region-I. Basic analysis. Int. J. Engng Sci. 14, 191-199, (1976).

[5] L. M. Brock, Symmetrical frictionless indentation over a uniformly expanding contact region-1I. Perfect adhesion. Int. J. Engng Sci. 15, 147-155, (1977).

[6] L. M. Brock, Dynamic analysis of nonsymmetric problems for frictionless indentation and plane crack extension. J. Elasticity. 8, 273-283. (1978).

[7] R. J. Tait \&T. B. Modde, Complex variable method and closed form solutions to dynamic crack and punch problem in classical theory of elasticity, I. J. Engineering Science, 19, 221-229, 1981.

[8] B. M. Singh \& R. S. Dhaliwal, Closed form solutions to dynamic problems by integral transformation method, $\mathrm{Z}$. Angew. Math. Mech, 64, 31-34, 1984.

[9] A. N. Das, Indentation of an elastic layer by four moving punches, Eng. Frac. Mech, 40(5), 815-823, 1993.

[10] Ravi Dalmeya, Ishan Sharma, Chandrashekhar Upadhyay,Akash Anand. Contact of a rigid cylindrical punch with an adhesive elastic layer. The Jour. Of Adhesion, 88,1-31,2012.

[11] Khan Zhou, Jen HsinHoh, Xu Wang, Leon M. Keer, H.L.John Peng, Bin Seng, Jem Q. Wang. A review of recent works on inclusions. Mech. Materials, 60, 144-158,2013.

[12] Chen Peijian, Chen Shaohua, Peng Juan. Sliding contact between a cylindrical punch and a graded halfplane with an arbitrary gradient direction. ASME., 82(4),041008(9 pages). 2015.

[13] I Ivan Argatov, JFederico Sabina. Small-scale indentation of an elastic coated half-space : The effect of compliant substrate. Int.J.Engng. Sci., 104, 87-96,2016

[14] Zdeněk Kala, "Limit States of Structures and Global Sensitivity Analysis Based on Cramér-von Mises Distance", International Journal of Mechanics, pp.107-118, Volume 14, 2020

[15] Diana Rubio, Domingo A. Tarzia, Guillermo F. Umbricht, "Heat Transfer Process with Solid-solid Interface: Analytical and Numerical Solutions", WSEAS Transactions on Mathematics, vol. 20, pp. 404-414, 2021

[16] I. G. Burova, "Application Local Polynomial and Non-polynomial Splines of the Third Order of Approximation for the Construction of the Numerical Solution of the Volterra Integral Equation of the Second Kind", WSEAS Transactions on Mathematics, vol. 20, pp. 9-23, 2021.

[17] I.Comez, M.A.Guler. On contact problem of a moving rigid cylindrical punch sliding over an orthotropic layer bonded to an isotropic half plane. Math and Mech. of solids. 25(10)1924-1942, 2020

[18] Ilyas Renreng, Muh. Farid Hidayat, Fauzan Djamaluddin, Crashworthiness Analysis of Octagonal-Inner Double Tube with different thickness under Off-Axis Oblique Load, International Journal of Mechanics, pp. 226-232, Volume 14, 2020

[19] Adel M. Al-Mahd ,On the Long-time Behavior of the Solution of a Non Linear Viscoelastic Plate Equation with infinite Memory and General Kernel, International Journal of Mechanics, pp. 141-150, Volume 14, 2020

\section{Creative Commons Attribution License 4.0 (Attribution 4.0 International, CC BY 4.0)}

This article is published under the terms of the Creative Commons Attribution License 4.0

https://creativecommons.org/licenses/by/4.0/deed.en_US 